\title{
A revolutionary use of wrist arthroscopy in paediatric arthrogryposis? a case report and review of the literature
}

Keywords: arthrogryposis, arthroscopy, wrist, mid-carpal wedge osteotomy, arthrogryposis, ligaments and joint capsules, avascular necrosis, laryngomalacia

\section{Introduction}

Arthrogryposis was first termed by Rosencrantz in 1905 to describe a curved joint ${ }^{1}$ with approximately $50 \%$ of patients having upper and lower limb involvement. ${ }^{2}$ Arthrogryposis manifests in a highly variable manner, with a classic presentation of the upper limb being that of a wasted shoulder, rigid elbow, flexed and ulnar deviated wrist and fingers with a thumb-in-palm deformity. ${ }^{3-7}$ Arthrogryposis of the wrist is complex and is often associated with dysplasia at the radiocarpal joint, further limiting movement in the wrist.4 Management of this involves addressing abnormalities of the joint and surrounding critical structures including abnormal insertions of muscles, tendons, ligaments and joint capsules. ${ }^{1,5}$ More recently, involvement of the triangular fibro-cartilage complex (TFCC) has also been noted in these patients. ${ }^{5}$

Conservative management of patients with arthrogryposis includes splinting, stretching and strengthening exercises. ${ }^{3,6}$ Surgical treatment aims to release tight structures by lengthening or shortening structures to place the wrist in a better position. ${ }^{3}$ These include combinations of mid-carpal wedge osteotomy, tendon transfers, volar fascia release, skin grafts and flaps and in severe cases, wrist fusion. ${ }^{1,4,7,8}$

To date, there are no cases reported in the literature of arthroscopic management of the arthrogrypotic wrist in either the adult or paediatric population. This minimally invasive approach has been used extensively for assessment and management of multiple wrist pathologies with few complications and an option to convert to an open procedure if necessary. We report here, the first, to our knowledge, case of a successful management of a painful wrist with stiffness from arthrogryposis via an arthroscopic approach.

\section{Case report}

A 16 years old female with distal arthrogryposis multiplex congenital presented with a stiff and painful left wrist with no preceding trauma. She had numerous medical comorbidities including myoclonic epilepsy, talipes, and developmental dysplasia of the left hip with avascular necrosis, laryngomalacia and anxiety. She had undergone multiple operative procedures for her other medical conditions, but had no operative intervention on her wrists. Her pain was primarily on the ulnar side of her wrist with limited use of her left hand for many activities of daily living. In particular, she presented with difficulties in brushing her hair and using cutlery. She avoided using her left hand and often tried to hide it as she did not like the look of its flexed position.

On examination, it was noted that her wrist rested at 50 degrees of flexion. The range of motion was limited passively to 0 degree of

\author{
Volume II Issue 6 - 2019
}

\author{
Kerstin Oestreich,' Andrea Jester,' Jeannette \\ Wen Ching Ting, I,2 Tommy R Lindau ${ }^{3}$ \\ 'Department of Plastic Surgery, Birmingham Women and \\ Children's Hospital, United Kingdom \\ ${ }^{2}$ Department of Plastic \& Reconstructive Surgery, Monash \\ Health, Australia \\ ${ }^{3}$ Pulvertaft Hand Centre, Derby, United Kingdom
}

\begin{abstract}
Correspondence: Kerstin Oestreich, Consultant Plastic Surgeon, Department of Plastic Surgery, Hand and Upper Limb Service, Birmingham Women and Children's Hospital, Steelhouse Lane, Birmingham, B4 6NH, United Kingdom, Tel +44I2I 333 8136, Email kerstin.oestreic@nhs.net
\end{abstract}

Received: November 14, 2019 | Published: November 29, 2019

extension and 80 degrees of flexion. All other ranges of motion were also very limited. On plain x-ray and MRI, it was noted that she had an ulnar plus variant and narrowing of the ulnocarpal joint space. It was suspected that she suffered from ulnar impaction syndrome with some TFCC pathology complicated by concomitant arthrogryposis. There were no signs of ankylosis of any of her carpal joints. Arthroscopy was done to further assess her left wrist joint. In general anaesthetic (GA) she was operated on in supine position with a hand table and a traction tower as per normal arthroscopy. The screen and technical equipment were at the foot-end.

Radio-carpal arthroscopy was done with the 3-4 (between the 3rd and 4th dorsal extensor compartments) portal as the viewing portal and the 4-5 (between the 4th and 5th dorsal extensor compartment) portal as the working portal. The joint was very difficult to scope due to the findings of a "snowstorm" of fibrotic plica bands (Figure 1), thickened synovium covering the TFCC (Figure 2) and further synovitis in the ulno-carpal recess (Figure 3). After debriding all these layers of tissues, the TFCC was found to be intact but stretched over the congenitally deformed ulnar head (Figure 4). There was also early stage chondromalacia of the lunate fossa of the radius.

At mid-carpal arthroscopy, we also found significant synovitis, fibrous plica bands, which were especially thickened in the fossa Poirier (in the palmar mid-carpal joint). This was all debrided. Postoperatively the patient reported a significant improvement in both hand function and position following the operation. At her one year follow up, she rated her pain relief as "significant". Her postoperative range of motion was 15 degrees of extension and 90 degrees of flexion. She was particularly pleased with the ability to be able to perform activities of daily life that she was not able to do pre-operatively such as doing her hair and using cutlery. 


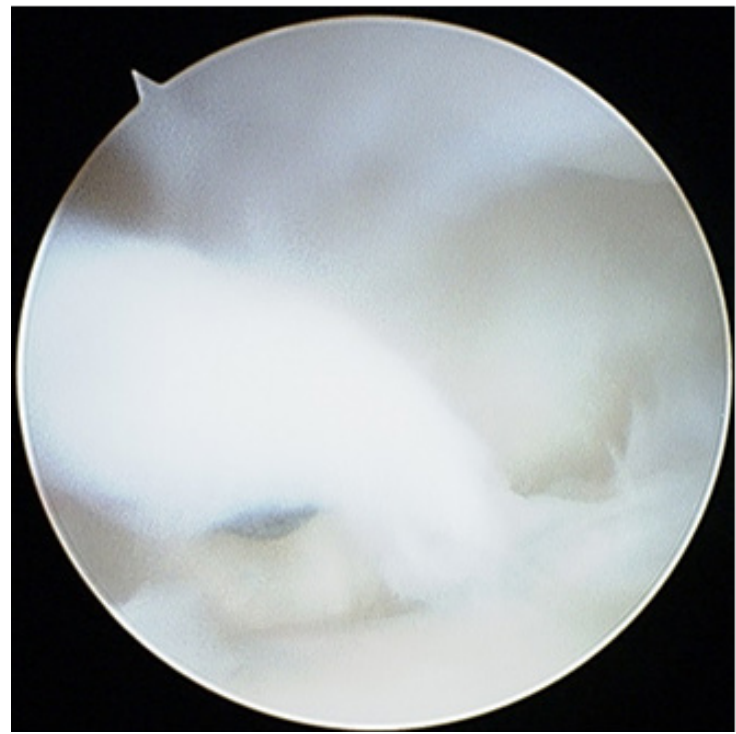

Figure I Left wrist radio-carpal arthroscopy (lunate on top, radius at the bottom) demonstrating the "snowstorm" of thick fibrous plica bands assessed with a probe (seen in the centre).

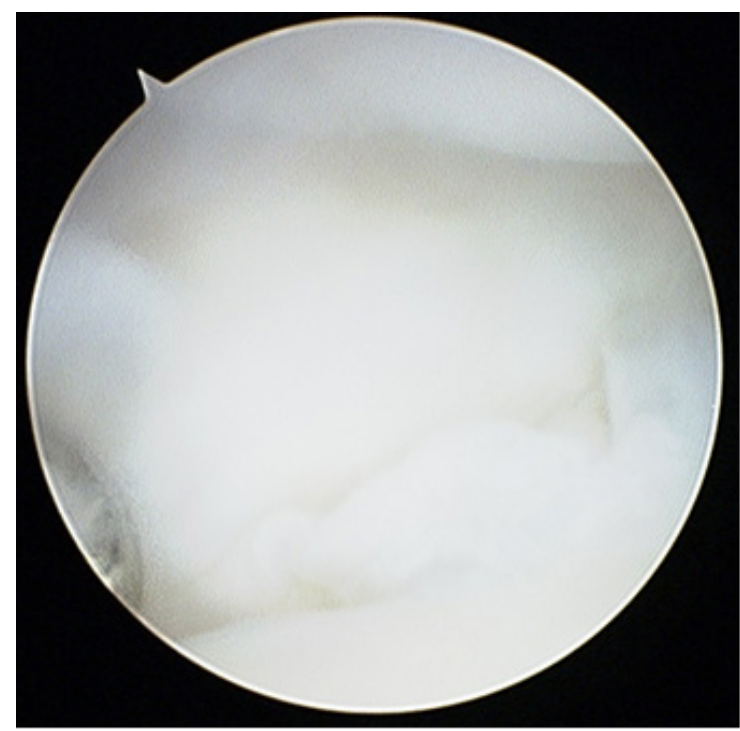

Figure 2 Radio-carpal arthroscopy left wrist (lunate on top, radius at the bottom) with thickened synovium covering the TFCC area in the ulno-carpal compartment seen once the plica bands have been excised.

\section{Literature review and integrated discussion}

To our knowledge, this case report describes the first case in the literature of an arthroscopically assisted arthrolysis of fibrous bands improving a paediatric patient's active and passive wrist range of movement in arthrogryposis. There was also a benefit seen in realigning the wrist from a flexed to a more neutral resting position whilst preserving radio-carpal movement. The cause of the extensive synovitis remained unclear, but the arthroscopy assisted synovectomy significantly improved her wrist pain and wrist motion. The aim of any treatment in patients with arthrogryposis involving the upper limb is to improve function; in our case we also managed to improve the pain.

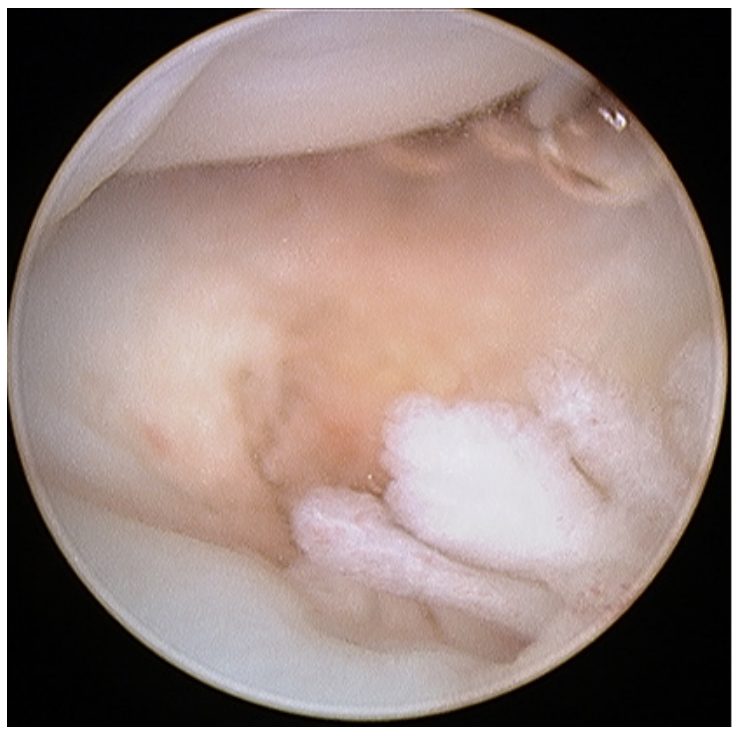

Figure 3 Radio-carpal arthroscopy left wrist (triquetrum on top, TFCC at bottom) after the plica bands and the thickened synovia has been removed, demonstrating further synovitis in the ulnocarpal recess.

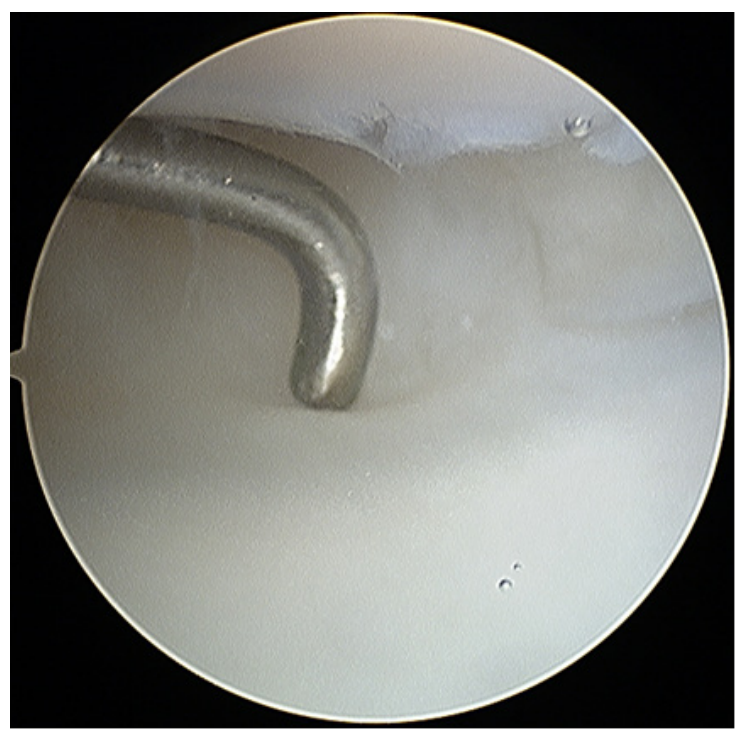

Figure 4 Radio-carpal arthroscopy left wrist (lunate on top, radius at the bottom) probe on the intact TFCC once the plica bands and thickened synovium have been debrided.

Non-surgical treatment of the wrist in patients with arthrogryposis typically involves intensive exercise program in the form of home stretching exercises. There is however, some evidence to suggest a variable benefit in improvement of movement in the wrist, especially when a child reaches three to four years of age where compliance is low. ${ }^{3}$ Splinting is another option but results vary due to difficulty with compliance and high recurrence rate. ${ }^{5,6}$ The literature suggests that this is presumably due to muscle imbalance once the splint is removed. ${ }^{3,6}$ Furthermore, it is believed that peri-articular structures are impossible to stretch and therefore, even without deforming forces from muscles, aggressive manipulation fails to increase range of motion in joints. ${ }^{7}$ Casting the wrist however, can help patients to 
decide if a planned wrist fusion position can improve the patient's function and should be trailed prior to such operations. ${ }^{3,7}$ One of the largest studies in the literature looking at serial casting results in 17 infants with upper limb arthrogryposis found that patients with distal arthrogryposis responded well to early serial casting whilst those with 'classic' arthrogryposis had poorer results and a recurrence rate of $75 \%{ }^{6}$ Our patient underwent extensive physiotherapy and serial casting as part of her management by a multi-disciplinary team with good compliance, but still requested additional intervention.

If the outcome of wrist arthroscopy in our patient had been poor the next surgical treatment option to address the pain and flexion deformity would have been to offer the patient a wrist fusion. The evidence of effectiveness of multiple tendon transfers, muscle lengthening or muscle detachment surgery or a combination of these operations ${ }^{1,2,3,5}$ is poor. Tendon lengthening and other tendon transfer procedures are said to be inadequate to improve movement and function due to strong deforming forces. ${ }^{1,4,5}$ In these cases, fusion with or without carpectomy has been described to be the main form of treatment in order to place the hand in a functional position. ${ }^{3,6-8}$ Not all studies agree that wrist fusion is the "gold standard" for patients with arthrogryposis and flexed wrists due to removal of any movement in the wrist further limiting what little function patients may have. Proximal row carpectomy is also not possible in the setting of mid-carpal coalition; a frequent occurrence in the wrist in patients with arthrogryposis over time4. Carpal wedge osteotomy is a popular technique amongst many surgeons as it places the wrist in a functional position and improves the appearance of the wrist. ${ }^{2,3,6-8}$ Most importantly, there is also a need to consider the status of the elbow and shoulder when performing these definitive surgeries on the wrist. ${ }^{3}$ Our patient understood that a wrist fusion may still be a treatment option for the future, if her wrist becomes more painful or she is not content with her wrist position.

Wrist arthroscopy might be an interesting technique to explore in both paediatric and adult arthrogrypotic patients. This treatment modality was introduced after the rationale that release of a joint capsule in stiff wrists can allow for greater movement, at least in adults, ${ }^{1}$ where 10-15 degrees of wrist extension was obtained from open release of the volar capsules. ${ }^{1}$ Arthroscopic joint release in adults has been useful if intra-articular structures are involved with wrist contractures; such as the radio-carpal septum and adhesive capsulitis of the wrist. ${ }^{9}$ There are to our knowledge no such procedures done with arthroscopy in the paediatric population.

The applications of wrist arthroscopy as such are not limited to non-trauma cohorts of patients. A study in adults recently described that arthroscopic excision of an intra-capsular post-traumatic radiocarpal septum can result in an increase in wrist motion by average of 22 degrees. ${ }^{9}$ Arthroscopic management of arthrofibrosis has been described in the literature for post-traumatic wrist stiffness in adults with good effect and minimal complications. ${ }^{10}$ The advantage of the arthroscopic technique is that it is a minimally invasive approach, whilst allowing for extensive release of capsular and ligamentous structures..$^{10}$ Instability of the carpus can be avoided if the palmar and ulnar ligaments are left intact with release of radio-scapho-capitate (RSC) and radio-lunate (SRL and LRL) ligaments. ${ }^{10}$

Moreover, interventions made by wrist arthroscopy, at least in the adult population, have been shown to be long lasting. Our patient maintained her improvement of her range of active motion after one year.

\section{Conclusion}

In this case report and literature review we describe the first case of an arthroscopic wrist intervention for arthrogryposis in a child. We have demonstrated that arthroscopic arthrolysis and synovectomy may greatly benefit the symptoms, both regarding increased mobility as well as pain. However, arthroscopy in this situation is extremely challenging and should be performed only by experienced wrist surgeons. Additional studies need to be conducted to further show the benefits of this technique in general, and in complex, multifactorial conditions such as arthrogryposis in particular.

\section{Acknowledgement}

None.

\section{Conflicts of interest}

The authors declare there is no conflict of interest.

\section{References}

1. Weeks PM. Surgical correction of upper extremity deformities in arthrogrypotics. Plast Reconstr Surg. 1965;36(4):459-465.

2. Oishi SN, Agranovich O, Pajardi GE, et al. Treatment of the upper extremity contracture/deformities. J Pediatr Orthop. 2017;37(5):S9S15.

3. Williams PF. Management of upper limb problems in arthrogryposis. Clin Orthop Relat Res. 1985;(194):60-67.

4. Ezaki M. An approach to the upper limb in arthrogryposis. J Pediatr Orthop. 2010;30(Suppl 2):57-62.

5. Bayne LG. Hand assessment and management of arthrogryposis multiplex congenita. Clin Orthop Relat Res. 1985;(194):68-73.

6. Smith DW, Drennan JC. Arthrogryposis wrist deformities: results of infantile serial casting. J Pediatr Orthop. 2002;22(1):44-47.

7. Hahn G. Arthrogryposis. Pediatric review and habilitative aspects. Clin Orthop Relat Res. 1985;(194):104-114.

8. Foy CA, Mills J, Wheeler L, et al. Long-term outcome following carpal wedge osteotomy in the arthrogrypotic patient. J Bone Jt Surg. 2013;95(20):e150.

9. Hattori T, Tsunoda K, Watanabe K, et al. Arthroscopic mobilization for contracture of the wrist. Arthroscopy. 2006;22(8):850-854.

10. Verhellen R, Bain GI. Arthroscopic capsular release for contracture of the wrist: a new technique. Arthroscopy. 2000;1978;16(1):106-110. 